\title{
Association Between Trust in Government and Practice of Preventive Measures During the COVID-19 Pandemic in Japan
}

\author{
Hiroshi Gotanda, MD, $P h D^{7}$, Atsushi Miyawaki, MD, $P h D^{2}$, Takahiro Tabuchi, $M D, P h D^{3}$, \\ and Yusuke Tsugawa, MD, $P h D^{4,5}$ (D)
}

\begin{abstract}
'Division of General Internal Medicine, Cedars-Sinai Medical Center, Los Angeles, CA, USA; 'Department of Public Health, Graduate School of Medicine, The University of Tokyo, Tokyo, Japan; ${ }^{3}$ Cancer Control Center, Osaka International Cancer Institute, Osaka, Japan; ${ }^{4}$ Division of General Internal Medicine and Health Services Research, David Geffen School of Medicine at UCLA, Los Angeles, CA, USA; ${ }^{5}$ Department of Health Policy and Management, UCLA Fielding School of Public Health, Los Angeles, CA, USA.
\end{abstract}

\begin{abstract}
BACKGROUND: Research suggests that preventive measures are critical to reducing the spread of coronavirus disease 2019 (COVID-19), but evidence regarding the association between trust in government and the practice of preventive measures is limited.
\end{abstract}

OBJECTIVE: To examine whether the practice of preventive measures against COVID-19 differs by one's level of trust in government.

DESIGN: A cross-sectional analysis using the Japan COVID-19 and Society Internet Survey (JACSIS) conducted in August and September 2020.

PARTICIPANTS: A nationally representative sample of Japanese individuals aged 15 through 79 years.

MAIN MEASURES: The primary outcome was the composite score for COVID-19 preventive measures, defined as the percentage of preventive measures an individual reported to be practicing (out of nine measures: social distancing, wearing masks, avoiding closed spaces, avoiding crowded spaces, avoiding close contact settings, hand washing, avoiding touching one's face, respiratory hygiene, and surface disinfection). The secondary outcomes were (1) support for stay-at-home requests, (2) use of a contact-tracing app, and (3) receipt of the influenza vaccine in the previous season.

KEY RESULTS: Our analysis included a total of 25,482 individuals. After adjusting for potential confounders, we found that individuals with high trust in government were likely to practice preventive measures more frequently compared to those with low trust (adjusted composite scores, $83.8 \%$ for high- vs. $79.5 \%$ for low-trust individuals; adjusted difference, +4.3 percentage points [pp]; $95 \%$ $\mathrm{CI},+2.4$ to $+6.2 \mathrm{pp} ; \mathrm{P}<0.001)$. We also found that high trust in government was associated with higher likelihoods of support for stay-at-home requests, use of a contact-tracing app, and receipt of the influenza vaccine in the previous season.

CONCLUSIONS: High trust in government was associated with a higher intensity of practicing COVID-19 preventive measures among Japanese individuals at the national

Hiroshi Gotanda and Atsushi Miyawaki contributed equally to this work.

Prior presentations: This study has never been presented.

Received January 20, 2021

Accepted May 28, 2021

Published online June 22, 2021 level. Our findings may provide useful information to develop and design effective public health interventions.

KEY WORDS: pandemic; coronavirus; COVID-19; trust in government.

J Gen Intern Med 36(11):3471-7

DOI: $10.1007 / \mathrm{s} 11606-021-06959-3$

(c) Society of General Internal Medicine 2021

\section{INTRODUCTION}

The outbreak of coronavirus disease 2019 (COVID-19) has led to a global public health emergency affecting tens of millions of people worldwide. Research has found that preventive measures - such as social distancing and maskwearing as recommended by the Centers for Disease Control and Prevention (CDC) and other health agencies - are critical to reducing the spread of COVID-19. ${ }^{1-5}$ While vaccines against COVID-19 are being developed and deployed at an unprecedented speed, non-pharmacological preventive measures will likely remain essential given the challenges to acquiring herd immunity including logistics and vaccine hesitancy. ${ }^{6}$ However, existing literature suggests that the adoption of such preventive measures is generally low, and there exist substantial variations across countries and regions. ${ }^{7-9}$ The underlying reasons why people do or do not choose to practice recommended preventive measures have not been well-characterized. This knowledge gap has hindered efforts to effectively control the transmission of COVID-19.

Studies of previous infection outbreaks suggest that trust in government is positively associated with preventive behaviors, ${ }^{10-12}$ but evidence is limited as to whether trust in government is an important contributing factor affecting the practice of preventive measures against COVID-19. A recent study conducted in China during the COVID-19 pandemic found that individuals with high trust in government were more likely to wear masks and wash their hands. ${ }^{13}$ Another study in Brazil using an online survey found that students with confidence in government were more likely to adopt preventive behaviors against COVID-19. ${ }^{14}$ An ecological study showed that among European countries, trust in politicians 
was associated with fewer non-essential activities. ${ }^{15}$ While informative, these studies are limited due to their small sample sizes $^{13,14}$ and focus on a limited number of preventive measures. $^{13,15}$ It is also unclear whether these findings were generalizable to other countries with different political and economic systems. ${ }^{13,14}$

Understanding the association between trust in government and the practice of preventive measures is critical for policymakers in many countries because recent work suggests a decline in trust in government after the outbreak of COVID19. ${ }^{16,17}$ This issue is particularly relevant in Japan as it does not have a national public health agency equivalent to the CDC in the USA, and many Japanese rely on the government as their main source of information about COVID-19. ${ }^{18}$ In addition, legal challenges make it difficult for the Japanese government to take strong executive actions including mask mandates and stay-at-home orders (a stay-at-home "request" was made on April 7, 2020, but violators faced no penalties). ${ }^{19}$

In this context, we examined the association between trust in government and the practice of preventive measures against COVID-19 using a large online survey conducted in August and September 2020 in Japan. We used preventive measures recommended by the Japanese government (that are very similar to the recommendations by the CDC), including social distancing, wearing masks, avoiding closed spaces, avoiding crowded spaces, avoiding close contact settings, hand washing, avoiding touching one's face, respiratory hygiene, and surface disinfection.

\section{METHODS}

\section{Data Source and Study Population}

We analyzed the data of Japanese individuals aged 15 through 79 years from the Japan COVID-19 and Society Internet Survey (JACSIS). JACSIS is a large internet survey conducted in August and September 2020 to examine how the COVID19 pandemic affected the daily lives of individuals in Japan. An invitation to participate in JACSIS was sent to the individuals in a large survey panel managed by a research agency with access to more than 2.3 million panelists. ${ }^{20}$ Individuals were invited using a stratified random sampling by age, gender, and prefecture category to reflect the national distribution of those attributes. A total of 224,389 invitations were sent based on the research agency's prior experience in achieving target sample sizes within survey periods. The survey was closed when the target numbers were reached: 28,000 participants in total and pre-specified sample sizes for each stratum by age, gender, and prefecture category. The survey participants accessed the designated website and provided information about their socioeconomic and physical and mental health statuses in the context of the COVID-19 pandemic, as well as their practice of preventive measures against COVID-19. The participants had the option of not responding to any part of the survey questionnaire and discontinuing the survey at any point.

We excluded $9.0 \%(\mathrm{n}=2518)$ of participants with unreliable responses based on the algorithm we developed. ${ }^{21}$ For example, we excluded participants who did not choose the correct answer to the following question: "Please choose the second choice from the bottom." The characteristics of the excluded sample were similar to the study sample.

\section{Exposure Variable}

Our exposure variable was the participants' level of trust in the government. Survey participants were asked to rate their agreement with the statement, "I trust the government," on a 4-point Likert scale. We defined that participants had "high" trust in government if they responded "Strongly Agree" or "Agree," and they had "low" trust in government if they responded "Disagree" or "Strongly Disagree" to this survey item.

\section{Outcome Variables}

Our primary outcome was the composite score for the practice of the nine COVID-19 preventive measures. The survey participants were asked to rate the frequency of performing nine different preventive measures (social distancing, wearing masks, avoiding closed spaces, avoiding crowded spaces, avoiding close contact settings, hand washing, avoiding touching one's face, respiratory hygiene, and surface disinfection) on a 4-point Likert scale (Always, Sometimes, Almost Never, Never) (Table 1). We defined the composite score for the practice of preventive measures as the percentage of items to which a participant responded "Always" or "Sometimes" out of nine items (i.e., the composite score ranges from 0 to $100 \%$ ). We also analyzed whether a participant responded "Always" or "Sometimes" to individual preventive measure items.

We additionally examined three preventive attitudes as secondary outcomes: (1) support for the stay-at-home request, (2) use of a contact-tracing app, and (3) receipt of the influenza vaccine in the previous season. We defined that participants supported stay-at-home requests if they responded "Strongly Agree" or "Agree" to the question asking whether the stay-athome request was appropriate.

\section{Adjustment Variables}

We included the following as individual-level adjustment variables in our multivariable regression models: age (categorized as $15-19,20-29, \ldots, 70-79$ ), gender, educational attainment (college or higher vs. high school or lower), income level categories using the tertiles of household equivalent income ("low" = less than 2.5 million Japanese Yen [JPY], "intermediate" $=2.5$ to 4.3 million JPY, and "high" = more than 4.3 million JPY), and an indicator of refusal to provide income information, household size (one, two, three, four, and five or 
Table 1 Description of the Nine COVID-19 Preventive Measures

\begin{tabular}{ll}
\hline \hline Preventive measure & Survey item (“Over the last month, how often did you...?”) \\
\hline Social distancing & Keep social distancing of $2 \mathrm{~m}$ (or $6 \mathrm{ft}$ ) \\
Wearing masks & Wear a mask when around other people \\
Avoiding closed spaces & Avoid closed spaces (e.g., by ventilating rooms) \\
Avoiding crowded spaces & Avoid crowded spaces \\
Avoiding close contact settings & Avoid close contact settings (e.g., talking loudly and shouting within 1m) \\
Hand washing & Wash your hands with soap and water for more than $15 \mathrm{~s}$ \\
Avoiding touching one's face & Avoid touching your eyes, nose, and mouth without washing your hands \\
Respiratory hygiene & Follow respiratory hygiene \\
Surface disinfection & Disinfect surfaces that are regularly touched (e.g., doorknobs) \\
\hline
\end{tabular}

The response options included "Always," "Sometimes," "Almost Never," and "Never" for each of the nine items. We defined the composite score for the preventive measure as the percentage of items to which a participant responded "Always" or "Sometimes" out of the nine items

more), marital status (married, never married, widowed, or separated), employment status (employer, self-employed, employee, or unemployed), smoking status (never, ever, or current), dummy variables for each of the eight comorbidities (overweight [body mass index $\geq 25 \mathrm{~kg} / \mathrm{m}^{2}$ ] and seven selfreported past medical histories of hypertension, diabetes, asthma, coronary artery disease, stroke, chronic obstructive pulmonary disease, and cancer), and dummy variables for 47 prefectures. We calculated the household equivalent income by dividing the gross (pre-tax) income in 2019 by the square root of the household size. We also calculated the body mass index by dividing self-reported body weight $(\mathrm{kg})$ by selfreported body height squared $\left(\mathrm{m}^{2}\right)$.

We included age, gender, household size, smoking status, and comorbidities as adjustment variables because participants with high risks for COVID-19 (e.g., older age, male gender, larger household size, currently smoking, more comorbidities) are expected to practice preventive measures more frequently. We also included educational attainment, income level, marital status, and employment status as these are known to be associated with health literacy, and those with high health literacy are likely to practice preventive measures.

\section{Statistical Analysis}

First, we compared the participants' characteristics between individuals with high trust in government versus those with low trust. We then examined the association between the participants' trust in government and their composite score for the COVID-19 preventive measures using a multivariable linear regression model, adjusting for potential confounders. We also investigated the associations between the participants' trust in government and each of nine individual preventive measures (included in the calculation of the composite score) and three secondary outcomes. We used multivariable linear regression models, instead of logistic regression models, for binary outcomes due to the ease of interpretation (i.e., linear probability models). ${ }^{22}$

We used inverse probability weighting (IPW) to account for the potential non-response bias and calculate national estimates. While our study population has distributions of age groups, sex, and prefecture categories similar to the national ones, it may not be nationally representative because those who responded to the survey may differ systematically from those who did not (e.g., access to the internet). Therefore, we estimated the probability of participation for each individual using an external dataset and used the inverse of these probabilities as weights (IPW) in our analyses. See Appendix Methods for more details.

We used the Benjamini-Hochberg method to account for the multiple comparisons across nine individual preventive measures and across three secondary outcomes, and report both unadjusted and adjusted $\mathrm{P}$ values (adjusted $\mathrm{P}$ values of less than 0.05 were considered to indicate statistical signifi(ance). ${ }^{23-25}$

\section{Sensitivity Analysis}

We conducted several sensitivity analyses. First, to test the sensitivity of our findings to the model specification, we reanalyzed the data with logistic regression models for binary outcomes (i.e., nine individual preventive measures and three secondary outcomes). Second, to examine whether our findings are sensitive to the definition of variables, we conducted sensitivity analyses by (i) defining participants with high trust in government as those who responded "Strongly Agree" to the trust question (as opposed to "Strongly Agree" or "Agree" in the main analysis) and (ii) defining the composite score for preventive measures as the percentage of items to which a participant responded "Always" to the preventive measure questions (as opposed to "Always" or "Sometimes" in the main analysis). Third, to evaluate whether our findings are sensitive to the potential non-response bias, we reanalyzed the data using unweighted regressions (not using the IPW method). Lastly, to examine whether the associations between trust in government and the practice of preventive measures vary by the characteristics of participants, we conducted stratified analyses by age (15-64 years vs. 65-79 years) and the presence of comorbidities (without comorbidities vs. with at least one comorbidity). In these stratified analyses, we first repeated the analyses for the stratified groups separately using the same models as the main analyses, and then estimated $\mathrm{P}$ values for the interaction terms between high trust in government and age 
group (or the presence of comorbidities) using the total sample to formally test the interaction terms.

All statistical analyses were performed using SAS version 9.4 (SAS Institute Inc., Cary, NC, USA). The study was reviewed and approved by the Research Ethics Committee of the Osaka International Cancer Institute (No. 20084).

\section{RESULTS}

This study included 25,482 individuals (mean [SD] age, 48.8 [17.4] years; 12,809 [50.3\%] female), of whom 10,572 $(41.5 \%)$ reported high trust in government and 14,910 (58.5\%) reported low trust in government (Table 2). The distributions of Likert scale responses to the questions on trust in government and the practice of preventive measures are shown in Appendix Figures 1 and 2.

\section{Primary Outcome}

After adjusting for potential confounders, we found that individuals with high trust in government were likely to practice preventive measures more frequently compared to those with low trust (83.8\% for high- vs. $79.5 \%$ for low-trust individuals; adjusted difference, +4.3 percentage points $[\mathrm{pp}] ; 95 \% \mathrm{CI},+2.4$ to $+6.2 \mathrm{pp} ; \mathrm{P}<0.001)($ Table 3$)$. Similar associations were observed across individual preventive measures - except for surface disinfection, for which we did not observe a statistically significant difference (Table 3 ). There was no evidence of collinearity among the variables included in the regression models.

\section{Secondary Outcomes}

We found that high trust in government was associated with higher likelihoods of support for the stay-at-home request (adjusted proportion, 93.2\% for high- vs. 66.9\% for low-trust individuals; adjusted difference, +26.3 percentage points [pp]; $95 \% \mathrm{CI},+23.9$ to $+28.6 \mathrm{pp} ; \mathrm{P}<0.001 ; \mathrm{P}<0.001$ after adjustment for multiple comparisons), use of a contact-tracing app (20.4\% vs. $14.6 \%$; adjusted difference, $+5.8 \mathrm{pp} ; 95 \% \mathrm{CI},+2.5$ to +9.1pp; $\mathrm{P}<0.001 ; \mathrm{P}<0.001$ after adjustment for multiple comparisons), and receipt of the influenza vaccine in the previous season $(37.7 \%$ vs. $32.2 \%$; adjusted difference, $+5.4 \mathrm{pp}$; $95 \%$ $\mathrm{CI},+1.7$ to $+9.1 \mathrm{pp} ; \mathrm{P}=0.005 ; \mathrm{P}=0.005$ after adjustment for multiple comparisons) (Table 4$)$.

\section{Sensitivity Analysis}

Our findings were qualitatively unaffected by using logistic regression models for binary outcomes (Appendix Tables 1 and 2). Analyses using an alternative definition of exposure and outcome variables showed results similar to the main analysis, although we observed a smaller effect size with an alternative definition of exposure variable and a larger effect size with an alternative definition of outcome variable (Appendix Tables 3 and 4). Unweighted analyses yielded very similar results to the main analysis (Appendix Table 5). A stratified analysis by age showed that individuals with high trust in government were practicing preventive measures more often than those with low trust among individuals aged 15-64 years $(\mathrm{P}<0.001)$, while this association was not observed among those aged 65-79 years $(\mathrm{P}=0.29)(\mathrm{P}-$ for-interaction $=$ 0.04) (Appendix Table 6). A stratified analysis by the presence of comorbidities showed that individuals with high trust in government were practicing preventive measures more often than those with low trust among both individuals without comorbidities $(\mathrm{P}<0.001)$ and those with comorbidities $(\mathrm{P}=$ $0.03)($ P-for-interaction $=0.08)($ Appendix Table 7$)$.

\section{DISCUSSION}

Using a large internet survey of more than 25,000 participants in Japan, we found that individuals with high trust in government practiced preventive measures against COVID-19 more often than those with low trust at the national level. We also found that trust in government was associated with broader preventive attitudes, such as support for stay-at-home requests. Our findings may be informative for policymakers in many countries who are struggling to implement preventive measures that have been recognized as cost-effective ways to mitigate the impact of the COVID-19 pandemic, given uncertainties about the COVID-19 vaccines.

The reason high trust in government is associated with practicing COVID-19 preventive measures can be extrapolated from the existing literature on public policy. Studies suggest that, when deciding whether to comply with public policies, people are equally concerned about the common good as they are about personal benefits. ${ }^{26,27}$ Individuals may be similarly more prepared to practice restrictive preventive measures when they trust the government and believe that it is working for the best interests of the population to curb the spread of COVID-19. While demographic, medical, and financial factors (e.g., age, comorbidities, income) likely play an important role in their decisions regarding the practice of preventive measures, our study indicated that the association between trust in government and the practice of preventive measures holds even after adjusting for these factors. In addition, our stratified analyses by age and comorbidities suggest that trust in government may have a stronger association with the practice of preventive measures among low-risk populations (e.g., younger individuals, those without comorbidities).

We did not observe a significant difference in the intensity of practicing surface disinfection between the two groups in the main analysis. Similarly, our stratified analysis by age suggested that older adults may be practicing surface disinfection less frequently than younger adults despite being at risk of contracting COVID-19. We hypothesize this is because the evidence of surface infection in preventing COVID-19 is weak and surface disinfection is not emphasized as strongly as other preventive measures by the Japanese government. ${ }^{28}$ 
Table 2 Characteristics of Participants According to Trust in Government

\begin{tabular}{|c|c|c|c|c|c|}
\hline \multicolumn{2}{|l|}{ Characteristics } & \multirow{2}{*}{$\begin{array}{l}\text { Total }(\mathbf{N}=\mathbf{2 5 , 4 8 2}) \\
12.809(50.3)\end{array}$} & \multirow{2}{*}{$\begin{array}{l}\text { High trust }(\mathbf{N}=\mathbf{1 0 , 5 7 2}) \\
5087(48.1)\end{array}$} & \multirow{2}{*}{$\begin{array}{l}\text { Low trust }(\mathbf{N}=\mathbf{1 4 , 9 1 0}) \\
7722(51.8)\end{array}$} & \multirow{2}{*}{$\frac{\text { P value }}{0.09}$} \\
\hline Female & & & & & \\
\hline Age, mean (SD), year & & $48.8(17.4)$ & $48.5(17.9)$ & $49.1(17.1)$ & 0.56 \\
\hline \multirow[t]{2}{*}{ Educational attainment } & College or higher & $12,701(49.8)$ & $5383(50.9)$ & 7318 (49.1) & \multirow[t]{2}{*}{0.40} \\
\hline & High school or lower & $12,781(50.2)$ & $5189(49.1)$ & $7592(50.9)$ & \\
\hline \multirow[t]{4}{*}{ Income level } & Lower & $7336(28.8)$ & $3061(29.0)$ & $4275(28.7)$ & \multirow[t]{4}{*}{0.10} \\
\hline & Intermediate & $6817(26.8)$ & $2716(25.7)$ & $4101(27.5)$ & \\
\hline & Higher & $5733(22.5)$ & $2636(24.9)$ & $3097(20.8)$ & \\
\hline & Not answered & $5595(22.0)$ & $2159(20.4)$ & $3436(23.0)$ & \\
\hline \multirow[t]{5}{*}{ Household size } & 1 & $4117(16.2)$ & 1543 (14.6) & $2573(17.3)$ & \multirow[t]{5}{*}{0.21} \\
\hline & 2 & $8574(33.7)$ & $3488(33.0)$ & $5086(34.1)$ & \\
\hline & 3 & $5927(23.3)$ & $2424(22.9)$ & $3503(23.5)$ & \\
\hline & 4 & $4352(17.1)$ & $1964(18.6)$ & $2388(16.0)$ & \\
\hline & $5+$ & $2513(9.9)$ & 1154 (10.9) & $1360(9.1)$ & \\
\hline \multirow[t]{4}{*}{ Marital status } & Married & $16,100(63.2)$ & $6942(65.7)$ & $9158(61.4)$ & \multirow[t]{4}{*}{0.37} \\
\hline & Never married & $6046(23.7)$ & $2325(22.0)$ & $3720(24.9)$ & \\
\hline & Widowed & $1949(7.7)$ & $838(7.9)$ & $1111(7.5)$ & \\
\hline & Separated & $1387(5.4)$ & 467 (4.4) & $920(6.2)$ & \\
\hline \multirow[t]{4}{*}{ Employment } & Employer & $1007(4.0)$ & $477(4.5)$ & $531(3.6)$ & \multirow[t]{4}{*}{0.84} \\
\hline & Self-employed & $2008(7.9)$ & $839(7.9)$ & $1169(7.8)$ & \\
\hline & Employee & $12,745(50.0)$ & $5275(49.9)$ & $7470(50.1)$ & \\
\hline & Unemployed & $9722(38.2)$ & $3982(37.7)$ & $5740(38.5)$ & \\
\hline \multirow[t]{3}{*}{ Smoking status } & Never & $12,959(50.9)$ & $5529(52.3)$ & $7430(49.8)$ & \multirow[t]{3}{*}{0.35} \\
\hline & Ever & $1638(30.0)$ & $2995(28.3)$ & $4643(31.1)$ & \\
\hline & Current & $4885(19.2)$ & $2048(19.4)$ & $2837(19.0)$ & \\
\hline \multirow[t]{8}{*}{ Comorbidities } & Overweight & $5185(20.4)$ & $2234(21.1)$ & $2952(19.8)$ & 0.42 \\
\hline & Hypertension & $6963(27.3)$ & $2803(26.5)$ & 4159 (27.9) & 0.56 \\
\hline & Diabetes & 2711 (10.6) & 1222 (11.6) & $1489(10.0)$ & 0.50 \\
\hline & Asthma & $3573(14.0)$ & $1520(14.4)$ & $2052(13.8)$ & 0.78 \\
\hline & Coronary artery disease & $1686(6.6)$ & $790(7.5)$ & $895(6.0)$ & 0.54 \\
\hline & Stroke & $1288(5.1)$ & $605(5.7)$ & $684(4.6)$ & 0.61 \\
\hline & COPD & $1103(4.3)$ & $501(4.7)$ & $603(4.0)$ & 0.77 \\
\hline & Cancer & $2185(8.6)$ & $915(8.7)$ & $1270(8.5)$ & 0.95 \\
\hline
\end{tabular}

The numbers are no. (\%), except for age. We used inverse probability weighting (IPW) for the probability of participating in our survey (see Appendix Methods for more details); therefore, the numbers of high-and low-trust individuals do not necessarily add up to the number of total participants. Income level indicates the tertiles of household equivalent income ("low" = less than 2.5 million Japanese Yen [JPY], "intermediate" = 2.5 to 4.3 million JPY, and "high" = more than 4.3 million JPY). COPD, chronic obstructive pulmonary disease; SD, standard deviation

We observed a substantially higher likelihood of support for stay-at-home requests among individuals with high trust in government compared to those with low trust (by 26.3 percentage points), perhaps reflecting the controversy over restricting economic activities despite the relatively low incidence of COVID-19 in Japan. While there were statistically significant differences in the use of a contact-tracing app and receipt of the influenza vaccine in the previous season by the level of trust in the government, the differences were small and may not be clinically meaningful. We found that less than $40 \%$ of individuals reported the receipt of the influenza vaccine in the previous season among both those with high and low trust in government. This vaccine hesitancy is likely derived from adverse events from vaccines in the past and the financial cost of receiving vaccines, ${ }^{29,30}$ and could be a challenge to achieving herd immunity against COVID-19 in Japan.

Our study was consistent with prior research that investigated the association between trust in government and the practice of preventive measures during the COVID-19 pandemic. A survey conducted in China $(n=3000)$ found that individuals with higher trust in government were more willing to wear masks and wash their hands. ${ }^{13}$ An online survey among 7554 students in the city of Sao Paulo, Brazil, also showed that confidence in government was associated with the adoption of 18 recommended preventive behaviors similar to those in our study for COVID-19. ${ }^{14}$ A country-level ecological study conducted in Europe reported that nonessential activities decreased more in countries with higher trust in politicians than in those with lower trust during the COVID-19 pandemic. ${ }^{15}$ While informative, existing studies have been limited in that they used a small sample size or specific population ${ }^{13,14}$ and examined a limited number of preventive measures. ${ }^{13,15}$ In addition, the findings might not be generalizable to countries with different political and economic systems. ${ }^{13,14}$ To our knowledge, our study is the first to examine the association between trust in government and the practice of preventive measures using a large national-level survey dataset with information on a broad set of preventive measures.

Our study has limitations. First, although we recognize the importance of preventive measures on controlling the spread of the virus, clinical implications of our observed differences in the use of preventive measures on the number of COVID-19 cases and deaths depend on the context, including the underlying incidence of the infections and whether preventive measures are enforced by law. Second, while we adjusted for potential confounders, it is still possible that there are unobserved variables - such as historical, cultural, or political factors - that can bias our estimates. Third, our study relied on self-reported data, which is subject to biases, such as recall bias and social desirability bias. Fourth, our study used the data from an internet survey, and specific groups in the 
Table 3 Association Between Trust in Government and Practice of Preventive Measures

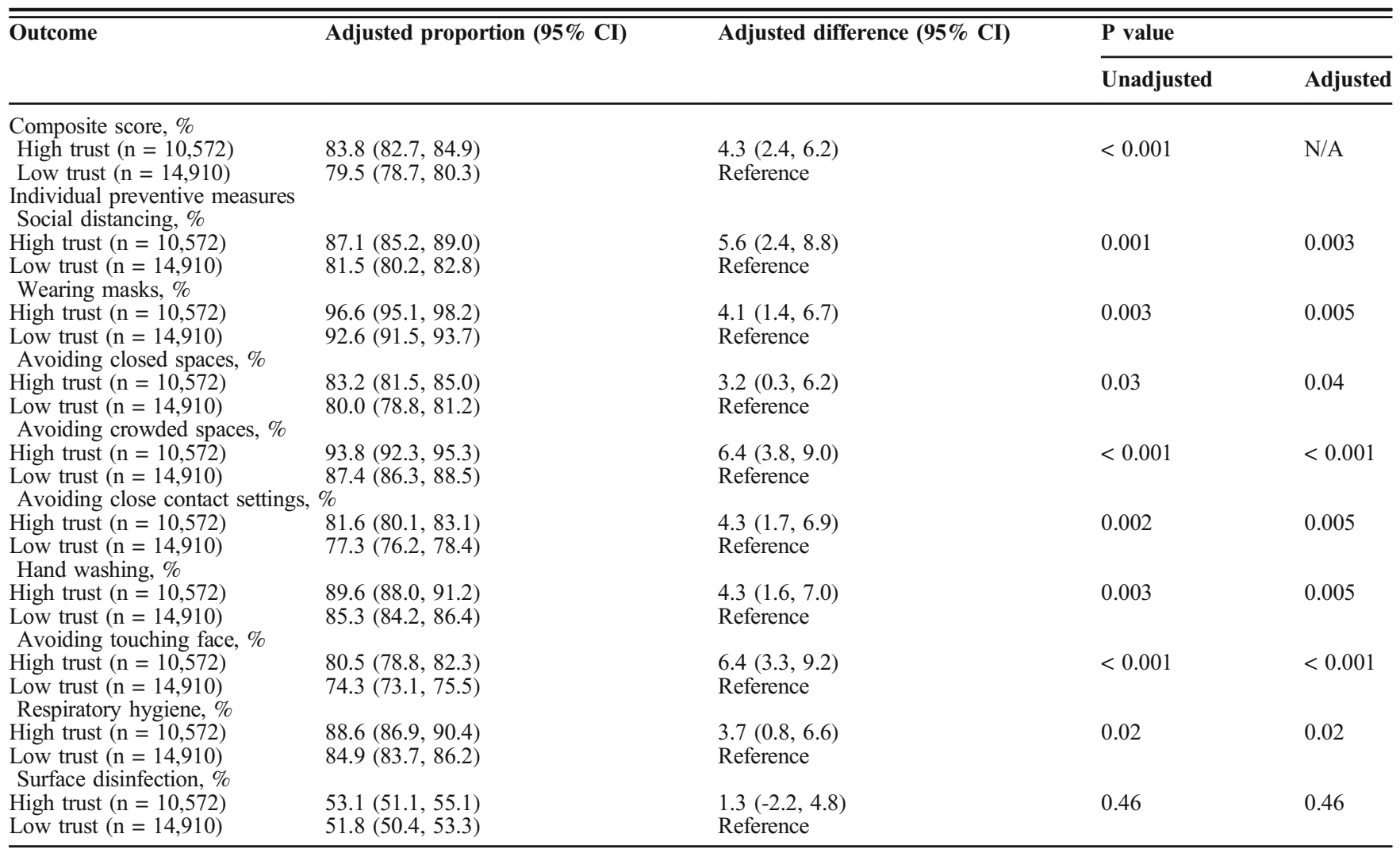

For each outcome, we constructed a weighted multivariable linear regression model, adjusting for individual-level characteristics (age, gender, educational attainment, income level categories, household size, marital status, employment status, smoking status, comorbidities, and prefectures). We used inverse probability weighting to account for the probability of participating in our survey (see Appendix Methods for more details). Adjusted proportions were calculated using marginal standardization. We used the Benjamini-Hochberg method to account for the multiple comparisons across nine outcomes (individual preventive measures). An adjusted $P$ value of less than 0.05 was considered statistically significant

population might be under-represented because of their limited access to the internet or other characteristics (i.e., nonresponse bias). To address this issue, we used stratified sampling to recruit a nationally representative sample and accounted for the probability of participating in our survey with weighted analyses. Lastly, our survey asked whether a participant trusts the government, and not the way the government handles the pandemic or the specific agency in charge of containing COVID-19. Therefore, it is possible that, while someone trusts the government in general, the person simultaneously does not trust the government's response to the pandemic and decides not to follow its guidance.

In summary, using data from a large internet survey in Japan conducted during the COVID-19 pandemic, we found that high trust in government is associated with a higher intensity of practicing preventive measures

Table 4 Association Between Trust in Government and Preventive Attitudes

\begin{tabular}{|c|c|c|c|c|}
\hline \multirow[t]{2}{*}{ Outcome } & \multirow[t]{2}{*}{ Adjusted proportion (95\% CI) } & \multirow[t]{2}{*}{ Adjusted difference (95\% CI) } & \multicolumn{2}{|l|}{$P$ value } \\
\hline & & & Unadjusted & Adjusted \\
\hline \multicolumn{5}{|c|}{ Support for stay-at-home requests, $\%$} \\
\hline $\begin{array}{l}\text { High trust } n=10,572 \text { ) } \\
\text { Low trust } n=14,910 \text { ) }\end{array}$ & $93.2(91.8,94.5)$ & $26.3(23.9,28.6)$ & $<0.001$ & $<0.001$ \\
\hline $\begin{array}{l}\text { Low trust } n=14,910 \text { ) } \\
\text { Use of contact-tracing ap }\end{array}$ & $66.9(65.9,67.9)$ & Reference & & \\
\hline \multicolumn{5}{|c|}{ Use of contact-tracing app, $\%$} \\
\hline High trust $\mathrm{n}=10,572$ ) & $20.4(18.5,22.3)$ & $5.8(2.5,9.1)$ & $<0.001$ & $<0.001$ \\
\hline Low trust $n=14,910$ ) & $14.6(13.2,16.0)$ & Reference & & \\
\hline \multicolumn{5}{|c|}{ Receipt of influenza vaccine in the previous season, \% } \\
\hline High trust $\mathrm{n}=10,572$ ) & $37.7(35.5,39.8)$ & $5.4(1.7,9.1)$ & 0.005 & 0.005 \\
\hline Low trust $n=14,910$ ) & $32.2(30.7,33.8)$ & Reference & & \\
\hline
\end{tabular}

For each outcome, we constructed a weighted multivariable linear regression model, adjusting for individual-level characteristics (age, gender, educational attainment, income level categories, household size, marital status, employment status, smoking status, comorbidities, and prefectures). We used inverse probability weighting to account for the probability of participating in our survey (see Appendix Methods for more details). Adjusted proportions were calculated using marginal standardization. We used the Benjamini-Hochberg method to account for the multiple comparisons across nine outcomes (individual preventive measures). An adjusted P value of less than 0.05 was considered statistically significant 
against COVID-19 at the national level. Our findings may provide useful information to policymakers who are struggling to control the spread of COVID-19 infections.

Supplementary Information The online version contains supplementary material available at https://doi.org/10.1007/s11606-02106959-3.

\section{Acknowledgements:}

Contributors: We would like to thank Drs. Kota Katanoda, Keisuke Kuwahara, Kanami Tsuno, Kenji Takeuchi, Hiroshi Murayama, AiHori, and Isao Muraki for the support related to the collection of the survey data.

Corresponding Author: Hiroshi Gotanda, MD, PhD; Division of General Internal Medicine, Cedars-Sinai Medical Center, Los Angeles, CA, USA (e-mail: ).

Funders This study was funded by the Japan Society for the Promotion of Science (JSPS) KAKENHI Grants (grant number 17H03589; 19K10671; 19K10446; 18H03107; 18H03062), the JSPS Grant-in-Aid for Young Scientists (grant number 19K19439), Research Support Program to Apply the Wisdom of the University to tackle COVID-19 Related Emergency Problems, University of Tsukuba, Health Labour Sciences Research Grant (grant number 19FA1005; 19FA1012; 19FG2001), and the Japan Agency for Medical Research and Development (AMED) (grant number: 2033648).

Data Availability The JACSIS study dataset is available upon request to the corresponding author.

\section{Declarations:}

Conflict of Interest: The authors declare that they do not have a conflict of interest.

Disclaimer: The findings and conclusions of this article are the sole responsibility of the authors and do not represent the official views of the research funders.

\section{REFERENCES}

1. World Health Organization 2020. Available at: https://www.who.int/ emergencies/diseases/novel-coronavirus-2019/advice-for-public. Accessed November 212020.

2. Centers for Medicare and Medicaid Services 2020. Available at: https:// www.cdc.gov/coronavirus/2019-ncov/prevent-getting-sick/prevention. html. Accessed November 232020.

3. Prem K, Liu Y, Russell TW, Kucharski AJ, Eggo RM, Davies N, et al. The effect of control strategies to reduce social mixing on outcomes of the COVID-19 epidemic in Wuhan, China: a modelling study. Lancet Public Health. 2020;5(5):e261-e70.

4. Schünemann HJ, Ak1 EA, Chou R, Chu DK, Loeb M, Lotfi T, et al. Use of facemasks during the COVID-19 pandemic. Lancet Respir Med. 2020;8(10):954-5.

5. Cochrane Library 2020. Available at: https://www.cochranelibrary.com/ collections/doi/SC000040/full. Accessed November 232020.

6. Fisher KA, Bloomstone SJ, Walder J, Crawford S, Fouayzi H, Mazor KM. Attitudes toward a potential SARS-CoV-2 vaccine: a survey of U.S. adults. Ann Intern Med. 2020.

7. Coroiu A, Moran C, Campbell T, Geller AC. Barriers and facilitators of adherence to social distancing recommendations during COVID-19 among a large international sample of adults. PLoS One. 2020;15(10):e0239795.
8. Al-Hasan A, Yim D, Khuntia J. Citizens' adherence to COVID-19 mitigation recommendations by the government: a 3-country comparative evaluation using web-based cross-sectional survey data. J Med Internet Res. 2020;22(8):e20634.

9. Geadan F, Akofua Mensah N, Tingey B, Bern R, Rees T, Talboys S, et al. What protective health measures are Americans taking in response to COVID-19? Results from the COVID Impact Survey. Int J Environ Res Public Health. 2020;17(17).

10. Gilles I, Bangerter A, Clémence A, Green EG, Krings F, Staerklé C, et al. Trust in medical organizations predicts pandemic (H1N1) 2009 vaccination behavior and perceived efficacy of protection measures in the Swiss public. Eur J Epidemiol. 2011;26(3):203-10.

11. Blair RA, Morse BS, Tsai LL. Public health and public trust: survey evidence from the Ebola Virus Disease epidemic in Liberia. Soc Sci Med. 2017; 172:89-97.

12. Vinck P, Pham PN, Bindu KK, Bedford J, Nilles EJ. Institutional trust and misinformation in the response to the 2018-19 Ebola outbreak in North Kivu, DR Congo: a population-based survey. Lancet Infect Dis. 2019;19(5):529-36.

13. Min C, Shen F, Yu W, Chu Y. The relationship between government trust and preventive behaviors during the COVID-19 pandemic in China: exploring the roles of knowledge and negative emotion. Prev Med. 2020;141:106288

14. Storopoli J, Braga da Silva Neto WL, Mesch GS. Confidence in social institutions, perceived vulnerability and the adoption of recommended protective behaviors in Brazil during the COVID-19 pandemic. Soc Sci Med. 2020:113477.

15. Bargain O, Aminjonov U. Trust and compliance to public health policies in times of COVID-19. J Public Econ. 2020;192:104316.

16. Pew Research Center 2020. Available at: https://www.pewresearch.org/ politics/2020/09/14/americans-views-of-government-low-trust-butsome-positive-performance-ratings/. Accessed December 12020.

17. Fancourt D, Steptoe A, Wright $\mathbf{L}$. The Cummings effect: politics, trust, and behaviours during the COVID-19 pandemic. Lancet. 2020;396(10249):464-5.

18. Japan Ministry of Internal Affairs and Communications 2020. Available at: https://www.soumu.go.jp/menu_news/s-news/01 kiban 18 01000082.html. Accessed March 122021.

19. Feldman EA 2020. Available at: https://www.theregreview.org/2020/ 06/10/feldman-japan-lenient-lockdown-conquer-coronavirus/. Accessed March 162021.

20. Rakuten Insight I 2020. Available at: https://insight.rakuten.co.jp/en/ aboutus.html. Accessed January 52021

21. Tabuchi T, Kiyohara K, Hoshino T, Bekki K, Inaba Y, Kunugita $\mathbf{N}$ Awareness and use of electronic cigarettes and heat-not-burn tobacco products in Japan. Addiction. 2016;111(4):706-13.

22. Hellevik $\mathbf{O}$. Linear versus logistic regression when the dependent variable is a dichotomy. Quality \& Quantity. 2009;43(1):59-74.

23. Benjamini Y, Hochberg $\mathbf{Y}$. Controlling the false discovery rate: a practical and powerful approach to multiple testing. J R Stat Soc B. 1995;57(1):289-300

24. Sentilhes L, Winer N, Azria E, Sénat M-V, Le Ray C, Vardon D, et al. Tranexamic acid for the prevention of blood loss after vaginal delivery. N Engl J Med. 2018;379(8):731-42.

25. Glickman ME, Rao SR, Schultz MR. False discovery rate control is a recommended alternative to Bonferroni-type adjustments in health studies. J Clin Epidemiol. 2014;67(8):850-7.

26. OECD 2013. Available at: https://www.oecd-ilibrary.org/content/component/gov glance-2013-6-en. Accessed December 302020

27. Murphy $\mathbf{K}$. The role of trust in nurturing compliance: a study of accused tax avoiders. Law and Human Behavior. 2004;28(2):187-209.

28. Ministry of Health Labour and Welfare 2020. Available at: https://www. mhlw.go.jp/stf/seisakunitsuite/bunya/0000164708_00079.html. Accessed January 132021.

29. Kuwabara N, Ching MSL. A review of factors affecting vaccine preventable disease in Japan. Hawai'i journal of medicine \& public health: a journal of Asia Pacific Medicine \& Public Health. 2014;73(12):376-81.

30. Tsukimori $\mathbf{O}$ 2020. Available at: https://www.japantimes.co.jp/news/ 2020/12/11/business/japan-slow-corona-vaccine/. Accessed December 312020.

Publisher's Note: Springer Nature remains neutral with regard to jurisdictional claims in published maps and institutional affiliations. 